\title{
The Application of Rough Sets Theory in Video Information Processing and Analysis
}

\author{
X.W. Li, Y.X. Kang \\ LanZhou Institute of Technology
}

\author{
X.W. Li \\ Key Laboratory of Gansu Advanced Control for Industrial \\ Processes
}

effective video data analysis tool and think to complete comprehensive computing. Aimed to solve this problem, the paper introduced the Rough Sets theory and applied to the video data analysis.

\section{BASIC THEORY AND PRINCIPLE OF ROUGH SETS}

In this section we will explore the most important theory and principle of Rough Sets used in this paper, especially the theory of Indiscernibility relation, Lower and Upper Approximations, Attributes Reduction and Core.

\section{A. Indiscernibility Relation}

Let $U \neq \phi$ be a universe of discourse and $\mathrm{X}$ be a subset of $U$. An equivalence relation, $\mathrm{R}$, classifies $U$ into a set of subsets $U / R=\left\{X 1, X 2, \ldots, X_{n}\right\}$ in which the following conditions are satisfied:

(1) $X_{i} \subseteq U, X_{i} \neq \phi$ For any i.

(2) $X_{i} \cap X_{j} \neq \phi \quad$ For any i, j.

(3) $\bigcup_{i=1,2, \ldots, n}, X_{i}=U$

Any subset $X_{i}$, which called a category, class or granule, represents an equivalence class of $\mathrm{R}$. A category in $\mathrm{R}$ containing an object $x \in U$ is denoted by ${ }^{[x]_{R}}$. For a family of equivalence relations $P \subseteq R$, an indiscernibility relation over $P$ is denoted by $I N D(P)$ and is defined by equation (1).

$$
\operatorname{IND}(P)=\bigcap_{R \in P} \operatorname{IND}(R)
$$

\section{B. Lower and Upper Approximations}

The set $X$ can be divided according to the basic sets of $R$, namely a lower approximation set and upper approximation set. Approximation is used to represent the roughness of the knowledge. Suppose a set $X \subseteq U$ represents a vague concept, then the R-lower and R-upper approximations of $X$ are defined by equation (2) and equation (3).

$$
\underline{R} X=\left\{x \in U:[x]_{R} \subseteq X\right\}
$$

Equation (4) is the subset of $X$, such that $X$ belongs to $X$ in $\mathrm{R}$, is the lower approximation of $\mathrm{X}$.

$$
\bar{R} X=\left\{x \in U:[x]_{R} \cap X \neq \phi\right\}
$$


Equation(5) is the subsets of all $\mathrm{X}$ that possibly belong to $\mathrm{X}$ in R, thereby meaning that $X$ may or may not belong to $\mathrm{X}$ in $R$, and the upper approximation $\bar{R}$ contains sets that are possibly included in X. R-positive, R-negative, and Rboundary regions of $\mathrm{X}$ are defined respectively by equation(4), equation(5) and equation(6).

$$
\begin{aligned}
& \operatorname{POS}_{R}(X)=\underline{R} X \\
& N E G_{R}(X)=U-\bar{R} X \\
& B N R(X)=\bar{R} X-\underline{R} X
\end{aligned}
$$

\section{Attributes Reduction and Core}

In RS theory, an Information Table is used for describing the object of universe, it consists of two dimensions, each row is an object, and each column is an attribute. RS classifies the attributes into two types according to their roles for Information Table: Core attributes and redundant attributes. Here, the minimum condition attribute set can be received, which is called reduction. One Information Table might have several different reductions simultaneously. The intersection of the reductions is the Core of the Information Table and the Core attribute are the important attribute that influences attribute classification.

A subset $B$ of a set of attributes $C$ is a reduction of $C$ with respect to $\mathrm{R}$ if and only if

(1)

$$
\begin{aligned}
& \operatorname{POS}_{B}(R)=\operatorname{POS}_{C}(R) \text {, and } \\
& \operatorname{POS}_{B-\{a\}}(R) \neq \operatorname{POS}_{C}(R) \text {, for any } a \in B
\end{aligned}
$$

And, the Core can be defined by equation (7)

$$
\operatorname{CORE}_{C}(R)=\left\{c \in C \mid \forall c \in C, \operatorname{POS}_{C-\left\{c_{\}}\right.}(R) \neq \operatorname{POS}_{C}(R)\right\}
$$

\section{THE APPLICATION OF ROUGH SETS IN VIDEO INFORMATION PROCESSING AND ANALYSIS}

\section{A. The Application of Rough Sets In Video Shots Detection}

The video is composed of many different video sequences frame, which is the most small units in video information. In shots detection, if we process every frame in video sequences, the efficiency of shots detection become very pool. So we must select the classic Representative frame to represent all video shots. In addition, as video exist many redundant data, before the analysis of video, we must determinate the impact of redundant data of video information. The novel data analysis tool RS can effective overcome the deficiency of above problem. The main think and steps can described as following.

Step1: extracting the DCT coefficients from original video sequences frame

Step2: extraction the DC coefficient from the DCT coefficients

Step3: constructing the information system using DC coefficients
Step4: divided the video shot using theory of Rough Sets attributes deduction.

We have made a lot of practical experiment use the method above to prove the feasibility and efficiency , figure 1 shows the final results of shots detection.
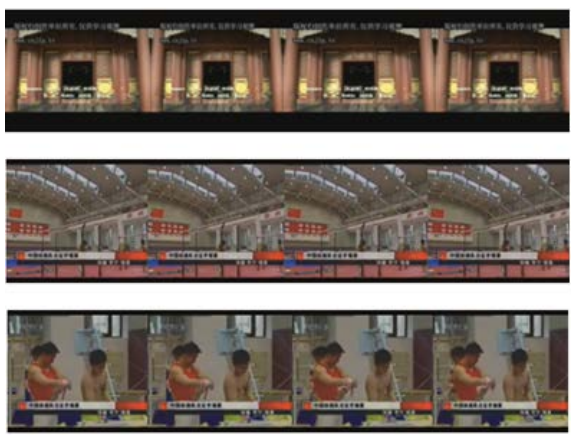

FIGURE I. THE FINAL RESULTS OF SHOTS DETECTION.

\section{B. The Application of Rough Sets In Key Frame Extraction}

Key frame is the Representative frame in video sequences, which is can represent the main video content produced from the original video sequences information, so in the video retrieval based on content, key frame is the first and crucial step in video retrieval and analysis. At present, the main problem in key frame extraction is large abundant video data, which can dramatically impact the whole system efficiency. While Rough Sets can effective omit the abundant data without need any pre-knowlege. based on above consideration, we can introduced the Rough Sets theory into key frame extraction techniques in video analysis. The main step can be described as following.

Step1: obtained the experience value of shots segmentation

Step2: extraction DCT coefficients and DC coefficients

Step3: constructing the information system using give DC coefficients

Step4: generating the core of information system

Step5: achieve the key frame of video

We also completed a serious of practical experiments used proposed algorithm to validated the feasibility and efficiency, the final results show that the developed algorithm can completed the object, figure 2 illustrate the results of key frame extraction.

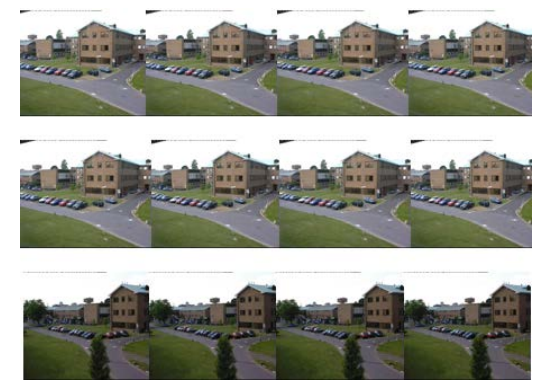

FIGURE II. THE FINAL RESULTS OF KEY FRAME EXTRACTION. 


\section{THE CONCLUSION AND PROSPECT}

Rough Sets is a novel and powerful tool for data analysis, it has successfully been used in many application domain, such as machine learning, expert system, digital image processing and pattern classification. the main advantage of Rough Sets theory is that it is does not need any preliminary or additional information about original data, like probability in statistics, or basic probability assignment in DempsterShafer theory and grade of membership or the value of possibility in fuzzy sets, especially, the attributes reduction theory of Rough Sets has widely used in many application domain. In this paper, we considered the Rough Sets theory application in video processing and analysis, especially exploring the application of shots detection and key frame extraction based on Rough Sets in compressed domain.

With the development of high and new technologies, especially the computer network technologies, more and more data will generate every second, Rough Sets will become a more important and necessary tool to many practical data analysis, for instance, to the digital video analysis or surveillance data.

\section{ACKNOWLEDGEMENTS}

The paper is supported by: (1) National Science Foundation project under grant NO.4661462057. (2)The Gansu construction scientific research projects of china under grant No:0612-05. (3)The Gansu scientific research project of universities under grant No.2013A-124. (4)The LanZhou special project of strategic new industry technology No.20134-23

\section{REFERENCES}

[1] SLOW INSKI R. Intelligent decision support2handbook of app licationsand advances of the Rough Sets theory [M ]. Dordrecht: luwer Aca2demic Publishers, 1992.

[2] PAWLAK Z. Rough Sets: theoretical aspects of reasoning about date[M ]. Dordrecht: Kluwer Publishers, 1991.

[3] PAWLAK Z, GRZYMALA2BUSSE J, SLOW INSKI R, et al. Rough Sets[ J ]. Commun ication of ACM, 1995, 38 (11): 89-95.

[4] PAWLAK Z. Rough Sets[ J ]. Interna tiona 1 Journa 1 of Informa tionand Computer Sc ience, 1982, 11 (5) : 341-356.

[5] Kin-Wai Sze, Kin-Man Lan Lam, and Guoping Qiu. a new key frame representation for video segment retrieval, IEEE Trans. Circuits and systems for technology, vol. 15, no. 9, pp. 1148-1155.2005.

[6] Sarah De Bruyne, Davy Van Deursen, and Jan De Cock, 2008. A comprssed-domain approach for shot boundary detection on H.264/AVC bit streams, singnal processing:image communication, vol.23, no.7, pp.473-489, 2008 .

[7] Aigrain P, Zhang H, and Petkovic D. Content-based representation and retrieval of visual media: a state-of-art review, multimedia tools and applications. Vol.3,no.3,pp.179-202,1996.

[8] Yang shuping, and Lin xinggang. Key Frame Extraction Using Unsupervised Clustering Based On a Statistical Model. Tsinghua science and technology. vol.10, no.2, pp.169-172, 2005.

[9] Man-Hee Lee, Hun-Woo Yoo, and Dong-Sik Jang, 2006. video scene change detection using neural network: improved ART2, Expert System with pplication. Vol. 31, pp.13-25.2006.

[10] Matthew Cooper, Ting Liu, and Eleanor Rieffel. video segmentation via temporal pattern classification, IEEE transactions on multimedia, vol.9, 2007. 